\title{
Quantum backaction in spinor-condensate magnetometry
}

\author{
S. K. Steinke, ${ }^{1,2}$ S. Singh,${ }^{3}$ P. Meystre, ${ }^{1}$ K. C. Schwab, ${ }^{2}$ and M. Vengalattore ${ }^{4}$ \\ ${ }^{1}$ B2 Institute, Department of Physics and College of Optical Sciences, The University of Arizona, Tucson, Arizona 85721, USA \\ ${ }^{2}$ Applied Physics, California Institute of Technology, MC 128-95, Pasadena, California 91125, USA \\ ${ }^{3}$ Institute for Theoretical Atomic, Molecular, and Optical Physics, Harvard-Smithsonian Center for Astrophysics, Cambridge, \\ Massachusetts 02138, USA \\ ${ }^{4}$ Laboratory of Atomic and Solid State Physics, Cornell University, Ithaca, New York 14853, USA \\ (Received 12 November 2012; revised manuscript received 26 August 2013; published 3 December 2013)
}

\begin{abstract}
We provide a theoretical treatment of the quantum backaction of Larmor frequency measurements on a spinor Bose-Einstein condensate by an off-resonant light field. Two main results are presented; the first is a "quantum jump" operator description that reflects the abrupt change in the spin state of the atoms when a single photon is counted at a photodiode. The second is the derivation of a conditional stochastic master equation relating the evolution of the condensate density matrix to the measurement record. We provide a few examples of the application of this formalism and comment on its application to metrology.
\end{abstract}

DOI: 10.1103/PhysRevA.88.063809

PACS number(s): 42.50.Lc, 07.55.Ge, 03.75.Gg

\section{INTRODUCTION}

Precision magnetometry has developed along several parallel tracks in recent years. Various magnetometers have been designed using diamond nitrogen vacancy (NV) centers $[1,2]$, with superconducting quantum interference devices representing a more established technology [3]. Atomic vapor magnetometers of spin-polarized alkali-metal atoms are another type of new field sensor with excellent performance [4]. These magnetometers, based on the optical detection of Larmor precession, have demonstrated field sensitivities in the aT/ $\mathrm{Hz}^{1 / 2}$ regime [5]. The use of optically trapped ultracold atoms as the sensing medium holds promise for magnetic microscopy at high spatial resolution as well as for significant improvements in field sensitivity via entanglementassisted techniques [6-8]. Spinor Bose-Einstein condensates are particularly suited to field sensing applications due to their low spin-relaxation rates and absence of density-dependent collision shifts [9].

The detection of Larmor precession and the subsequent estimation of the magnetic field rely on the dispersive interaction between the collective atomic spin and the optical field, followed by a quantum-limited measurement of the light. This interaction entangles the optical and atomic degrees of freedom. Measuring the light breaks this entanglement and must therefore cause a backaction on the atomic spins.

The ultimate sensitivity of such atomic magnetometers is governed by the interplay between the projection noise of the atomic spins, photon shot noise, and quantum backaction due to the measurement of the optical field. Here, we provide a theoretical treatment of this backaction by means of a conditional stochastic master equation that relates the condensate evolution to the optical measurement record. One of the main results that distinguishes this work from similar studies, e.g., Ref. [10,11], is the derivation of a quantum jump operator reflecting the backaction on the atoms induced by the detection of a single photon.

This paper is organized as follows. Section II introduces our model of Larmor precession measurements via photoelectron counting in a balanced Mach-Zehnder interferometer, discussing in general terms the photocount probabilities and the measurement-reduced state of the atomic system. Section III then introduces the quantum jump operators appropriate for detection with feeble laser fields, and Sec. IV applies these results to the development of a conditional stochastic equation that describes the measurement scheme and its backaction on the atomic system. Section V applies this formalism to a couple of illustrative examples, and finally Sec. VI is a summary and outlook.

\section{MODEL}

We consider a magnetometer consisting of a spinor BoseEinstein condensate of $n \gg 1$ spin-1 bosons trapped in their motional ground state. The basic idea is that any external magnetic field $\vec{B}(t)$ will modify the Larmor precession frequency of the spins, so that measuring that frequency is a measurement of $\vec{B}$.

The formalism that we develop in the following is well suited to both spinor gases with a ferromagnetic ground state, such as ${ }^{87} \mathrm{Rb}$, and those with a polar ground state, such as ${ }^{23} \mathrm{Na}$. It can also be readily extended to systems with different internal state spaces or expanded to incorporate spatial variations in the atomic spin field, although we assume in this paper that the spatial extent of the gas is small enough that the single-mode description of its center of mass is appropriate.

Before proceeding further, we introduce multi-index notation for the spin indices. This serves two purposes: first, it shortens otherwise lengthy equations, making them easier to read and parse; more importantly, it allows our general results to be applied directly to atomic systems with different internal manifolds simply by changing the number of indices as appropriate. Specifically, we define

$$
\begin{gathered}
\mathbf{v}=\left(v_{+}, v_{0}, v_{-}\right), \\
|\mathbf{v}|_{1}=v_{+}+v_{0}+v_{-}, \\
\mathbf{v} !=v_{+} ! v_{0} ! v_{-} ! \\
\mathbf{v}^{\mathbf{w}}=v_{+}^{w_{+}} v_{0}^{w_{0}} v_{-}^{w_{-}}, \\
\mathbf{v} \cdot \mathbf{w}=v_{+} w_{+}+v_{0} w_{0}+v_{-} w_{-} .
\end{gathered}
$$


We use the one-norm rather than the more common two-norm in Eq. (2) to make summation indices more elegant. The notation in Eqs. (3) and (4) commonly appears in multinomial arithmetic and multivariate calculus. Lastly, the dot product notation in Eq. (5) is, aside from the spin rather than coordinate indices, the same as it would be for spatial vectors, which are denoted with over-arrows in this work to avoid ambiguity.

The atoms are prepared initially with high fidelity in the pure spin state:

$$
|\psi\rangle=c_{+}|+1\rangle+c_{0}|0\rangle+c_{-}|-1\rangle,
$$

for example, in the $z$ basis. Under the influence of a transverse magnetic field these coefficients will become time dependent.

For later use, we define a probability multi-index:

$$
p_{i}=\left|c_{i}\right|^{2},
$$

where $i \in\{+, 0,-\}$, with the state normalization implying that $|\mathbf{p}|_{1}=1$. Single-atom spin expectation values are given in terms of $p_{i}$ as

$$
\begin{aligned}
& \left\langle F_{z}\right\rangle=p_{-}-p_{-}, \\
& \left\langle F_{z}^{2}\right\rangle=p_{+}+p_{-} .
\end{aligned}
$$

With this notation, the full $n$-particle state can be compactly expressed as

$$
\left|\psi_{n}\right\rangle=\hat{a}_{\psi}^{\dagger n}|0\rangle / \sqrt{n !}=\sum_{|\mathbf{j}|_{1}=n} \sqrt{\frac{n !}{\mathbf{j} !}} \mathbf{j}|\mathbf{j}\rangle,
$$

where $\hat{a}_{\psi}^{\dagger}=\mathbf{c} \cdot \hat{\mathbf{a}}^{\dagger}$ is the Schrödinger field creation operator for state $|\psi\rangle,|0\rangle$ is the vacuum, and $|\mathbf{j}\rangle$ is the state with $j_{i}$ atoms in spin eigenstate $i$.

The spin state of the condensate is optically detected either by measuring the phase imprinted onto a coherent polarized pulse of the light field by the condensate, as in phase-contrast imaging [12,13], or by monitoring the polarization of the light field, as in polarization spectroscopy (see, for example, [14]). Without loss of generality, we assume the former method via balanced homodyne detection. The condensate sits in one arm of a balanced Mach-Zehnder interferometer, and the phase shift is measured by subtracting the photocurrents at the two detectors (see Fig. 1).

We assume that the probe field is detuned sufficiently far from the atomic resonance so that the excited atomic states can be adiabatically eliminated. We also assume an optical transit time short enough that other contributions to the condensate's evolution may be neglected for now. By introducing a multi-index of coupling strengths $\mathbf{g}$ that reflects the spin dependence of the light-atom interaction, we then find the interaction Hamiltonian:

$$
H_{I}=\hbar \mathbf{g} \cdot \hat{\mathbf{N}} \hat{b}^{\dagger} \hat{b}
$$

where $N_{i}=\hat{a}_{i}^{\dagger} \hat{a}_{i}$ and $\hat{b}$ is the light field annihilation operator. See the Appendix for a detailed derivation in a specific example. The effect of this Hamiltonian is to imprint a phase rotation on the optical field that contains information about the spin state of the atoms.

The quantum state of the combined atomic spin and two output optical fields after passing through the final beam

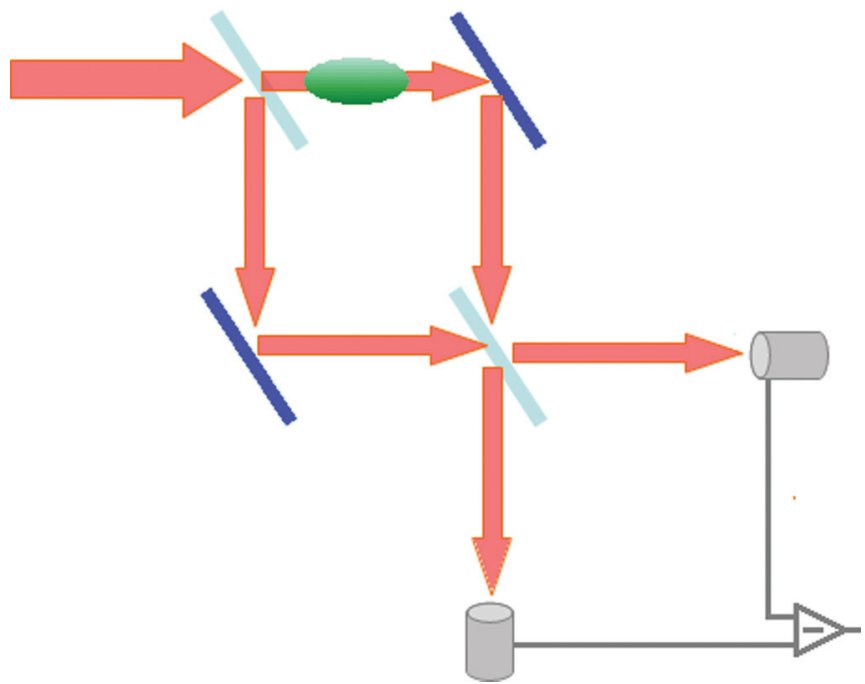

FIG. 1. (Color online) A schematic of the proposed experimental setup. The atomic condensate sits in one arm of a balanced MachZehnder interferometer, where two 50:50 beam splitters are adjusted such that the output light will have equal intensity at each port in the absence of any material inducing an additional phase shift. Thus, the phase shift the atoms imprint on the light can be determined by measuring the differential signal from the photodiodes.

splitter, just before measurement, is therefore

$$
\begin{aligned}
|\psi\rangle= & \sum_{|\mathbf{j}|_{1}=n} \sqrt{\frac{n !}{\mathbf{j} !}} \mathbf{c}^{\mathbf{j}}|\mathbf{j}\rangle \otimes\left|\frac{A_{0}}{2}\left(1+i e^{-i \mathbf{G} \cdot \mathbf{j}}\right)\right\rangle \\
& \otimes\left|\frac{A_{0}}{2}\left(1-i e^{-i \mathbf{G} \cdot \mathbf{j}}\right)\right\rangle,
\end{aligned}
$$

where $A_{0}$ is the dimensionless amplitude of the coherent optical pulse into the first beam splitter so that $\left\langle\hat{b}^{\dagger} \hat{b}\right\rangle=\left|A_{0}\right|^{2} / 2$, $\tau$ is the light-matter interaction time, and the single-photon single-atom dimensionless coupling is $\mathbf{G}=\tau \mathbf{g}$.

In deriving Eq. (11) we have assumed that no light is lost at the mirrors or beam splitters and that absorption by the atomic sample is likewise negligible, so that the output light fields may be expressed in terms of their coherent amplitudes. We also note that the additional phase rotations due to the free propagation of the light cancel out if the interferometer arms are properly balanced. The probe beam's contribution to the output fields has been phase rotated due to the unitary evolution induced by the Hamiltonian Eq. (10). Importantly, unless the atoms are in the unlikely configuration of a Fock state of the spin field, this interaction entangles the optical field with the collective spin state of the atoms [15]. In the rest of this article, we quantify the backaction induced on the condensate due to measurement of the light.

Assuming the photodetectors have unit efficiency, the joint probability of obtaining photocounts $C_{+}$and $C_{-}$at the first and second photodiodes, respectively, is

$$
\begin{aligned}
P\left(C_{+}, C_{-}\right)= & e^{-\left|A_{0}\right|^{2}} \frac{\left(\left|A_{0}\right|^{2} / 2\right)^{C_{+}+C_{-}}}{C_{+} ! C_{-} !} \sum_{|\mathbf{j}|_{1}=n} \frac{n !}{\mathbf{j} !} \mathbf{p}^{\mathbf{j}} \\
& \times(1+\sin \mathbf{G} \cdot \mathbf{j})^{C_{+}}(1-\sin \mathbf{G} \cdot \mathbf{j})^{C_{-}} .
\end{aligned}
$$


After an ideal measurement with outcome $\left(C_{+}, C_{-}\right)$the atomic state is given by

$$
\begin{aligned}
\left|\psi\left(C_{+}, C_{-}\right)\right\rangle= & \mathcal{N} \sum_{|\mathbf{j}|_{1}=n} \sqrt{\frac{n !}{\mathbf{j} !}} \mathbf{c}^{\mathbf{j}}\left(1+i e^{-i \mathbf{G} \cdot \mathbf{j}}\right)^{C_{+}} \\
& \times\left(1-i e^{-i \mathbf{G} \cdot \mathbf{j}}\right)^{C_{-}}|\mathbf{j}\rangle,
\end{aligned}
$$

where $\mathcal{N}$ is the normalization factor. Note that this state can in general no longer be expressed as $n$ atoms in an identical single particle state as in Eq. (9).

To proceed, we make the assumption that $n|\mathbf{G}| \ll 1$. Physically this implies weak coupling between a single photon and the condensate, or equivalently it means that the phase rotation imprinted on the light is small. This is typically the case for off-resonant light, as the coupling constants $\mathbf{G}$ are inversely proportional to the detuning and directly proportional to the light-atom interaction time. For a condensate that is micron scale, this time is on the order of tens of femtoseconds. Of course, to measure a very small phase rotation in turn requires a large incident photon number, that is, intense light. Dephasing of the condensate due to optical absorption thus may be a concern. However, this effect scales inversely with the detuning squared. On the other hand, the off-resonant coupling and, hence, the signal scale only inversely with the detuning. This yields the important result that by increasing both the detuning and the intensity one can amplify the signal while suppressing absorption by the atomic state.

Using Eqs. (12) and (8) and with the above assumption, we find for the mean observed photon count difference the expression

$$
\begin{aligned}
\langle\Delta C\rangle= & \sum_{C_{+}, C_{-}} P\left(C_{+}, C_{-}\right)\left(C_{+}-C_{-}\right) \approx\left|A_{0}\right|^{2} n \mathbf{G} \cdot \mathbf{p} \\
= & \left|A_{0}\right|^{2} n\left(G_{0}+\frac{G_{+}-G_{-}}{2}\left\langle F_{z}\right\rangle\right. \\
& \left.+\frac{G_{+}-2 G_{0}+G_{-}}{2}\left\langle F_{z}^{2}\right\rangle\right) .
\end{aligned}
$$

The signal in Eq. (14) can be interpreted as follows. If the atoms are precessing in a magnetic field perpendicular to the direction of propagation of the light field, the phase contrast signal has a large dc component proportional to the number of atoms, an ac signal at the Larmor precession frequency, and a third smaller signal at twice the Larmor frequency. The magnitude of the respective contributions can be controlled by changing the polarization and/or the detuning of the probe light, thereby changing the relative values of the quantities G [12].

Finally, since in an experiment the number of condensed atoms is classically uncertain the spin state $\left|\Psi_{n}\right\rangle$ must be replaced by a density matrix $\hat{\rho}$. The atom number in an experimental condensate obeys roughly Poissonian statistics:

$$
\hat{\rho}=e^{-\bar{n}} \sum_{n=0}^{\infty} \frac{\bar{n}^{n}}{n !}\left|\psi_{n}\right\rangle\left\langle\psi_{n}\right|,
$$

where $\bar{n}$ is the mean atom number over many runs and $\left|\Psi_{n}\right\rangle$ is given by Eq. (9). Repeating the computation in Eq. (12) in this case leads to a simple modification of Eq. (14): the substitution $n \rightarrow \bar{n}$. Similarly, the postmeasurement density matrix is found by substituting the state in Eq. (13) into Eq. (15), with a different overall normalization factor.

\section{QUANTUM JUMP OPERATOR}

So far, we have considered the measurement of the condensate state by a pulse of light. To reach a continuous measurement limit, we consider coherent light with a photon flux per unit time $f$ and examine the evolution of the system in a time interval $\delta t$ such that $f \delta t \ll 1$. By making the substitution $A_{0} \rightarrow \sqrt{f \delta t}$ we model this continuous observation as a series of short, weak pulses. In this case, the probability for detecting $C=C_{+}+C_{-}$total photons in a single "pulse" is proportional to $(f \delta t)^{C}$. Therefore, to leading order, we need only consider the cases where zero or one photons are detected. The free evolution of the atoms is negligible on this timescale, i.e., between individual photon clicks.

Rather than restricting ourselves to the case where there are $n$ atoms in an identical spin state, as in the previous section, we consider an arbitrary initial atomic state:

$$
\left|\psi_{\mathrm{I}}\right\rangle=\sum_{\mathbf{j}} c_{\mathbf{j}}|\mathbf{j}\rangle .
$$

To leading order in $f \delta t$, the postmeasurement states for the three outcomes with zero or one total photons are given by a slight modification of Eq. (13):

$$
\begin{aligned}
& |\psi(0,0)\rangle=\left(1-\frac{f \delta t}{2}\right)\left|\psi_{\mathrm{I}}\right\rangle, \\
& |\psi(1,0)\rangle=\frac{\sqrt{f \delta t}}{2} \sum_{\mathbf{j}} c_{\mathbf{j}}\left(1+i e^{-i \mathbf{G} \cdot \mathbf{j}}\right)|\mathbf{j}\rangle, \\
& |\psi(0,1)\rangle=\frac{\sqrt{f \delta t}}{2} \sum_{\mathbf{j}} c_{\mathbf{j}}\left(1-i e^{-i \mathbf{G} \cdot \mathbf{j}}\right)|\mathbf{j}\rangle,
\end{aligned}
$$

where these are normalized to reflect the respective probabilities of their occurrence, which are, to leading order,

$$
\begin{aligned}
& P(0,0)=1-f \delta t, \\
& P(1,0)=\frac{f \delta t}{2}\left(1+\sum_{\mathbf{j}}\left|c_{\mathbf{j}}\right|^{2} \sin \mathbf{G} \cdot \mathbf{j}\right), \\
& P(0,1)=\frac{f \delta t}{2}\left(1-\sum_{\mathbf{j}}\left|c_{\mathbf{j}}\right|^{2} \sin \mathbf{G} \cdot \mathbf{j}\right) .
\end{aligned}
$$

From Eqs. (17) we see that a single click has the effect of acting on the state with quantum jump operators

$$
\begin{aligned}
|\psi(1,0)\rangle & =\hat{J}_{+}\left|\psi_{\mathrm{I}}\right\rangle \\
|\psi(0,1)\rangle & =\hat{J}_{-}\left|\psi_{\mathrm{I}}\right\rangle \\
\hat{J}_{ \pm} & =\frac{\sqrt{f \delta t}}{2}\left(1 \pm i e^{-i \mathbf{G} \cdot \hat{\mathbf{N}}}\right),
\end{aligned}
$$

and "no click" simply rescales the state slightly.

The same analysis applied to an arbitrary initial density matrix yields a similar result; namely, a single click at one of the detectors acts on the density matrix with one of the jump operators $\hat{J}_{ \pm}$. For example, in the standard theory 
of photodetection-a one-sided cavity with damping rate $\kappa$ and single mode operator $\hat{a}$-the detection of a photon is characterized by the jump operator $\sqrt{\kappa \delta t} \hat{a}$ [16]. While this appears to be similar to the present case, a difference is that the light field is now used to determine the dynamics of the atoms, but no atoms are annihilated in the process. Also, as long as $\langle\mathbf{G} \cdot \hat{\mathbf{N}}\rangle \ll 1$, a single jump will have only a very small effect on the atomic state. This is to be expected-a single nonresonant photon passing through our apparatus ought not perturb the atoms much. In the next section, we combine this jump operator with the free spin evolution of the atoms to arrive at a master equation describing the effects of the detection process.

\section{CONDITIONAL STOCHASTIC MASTER EQUATION}

With these results in hand we are now in a position to derive a stochastic master equation that also includes the effect of an external, possibly time-dependent magnetic field $\vec{B}(t)-$ eventually the field to be detected-on the spin dynamics. Consistent with the experimental situation, we consider both the light-atom interaction and the Zeeman interaction induced by $\vec{B}$ :

$$
H_{\mathrm{Z}}=\hbar g_{\mathrm{L}} \mu_{\mathrm{B}} \vec{B} \cdot \hat{\vec{F}},
$$

where $g_{\mathrm{L}}$ is the Landé $g$ factor, $\mu_{\mathrm{B}}$ is the Bohr magneton, and $\hat{\vec{F}}$ are the Schrödinger field spin operators, e.g., $\hat{F}_{z}=$ $\hat{N}_{+}-\hat{N}_{-}$. This Hamiltonian could be modified or replaced in more general settings, for instance, by including the quadratic Zeeman effect. We consider the dynamics of the system over a time scale $\Delta t \gg \delta t$ and an incident flux $f$ such that a natural separation of scales occurs:

$$
\begin{gathered}
f \Delta t \sim \epsilon^{-1}, \\
g_{\mathrm{L}} \mu_{\mathrm{B}}\langle\vec{B} \cdot \hat{\vec{F}}\rangle \Delta t \sim \epsilon, \\
\langle\mathbf{G} \cdot \hat{\mathbf{N}}\rangle \sim \epsilon^{2} .
\end{gathered}
$$

Roughly speaking, these scales establish a large photon number, a small free evolution, and an even smaller effect from a single quantum jump, respectively. However, in the time interval $\Delta t$ there will be order $\epsilon^{-1}$ jumps; hence, the free evolution and measurement induced dynamics will be of comparable order.

Next, we consider the set of all possible photocounts $\left\{\left(C_{+}, C_{-}\right)\right\}$and the probabilities of their detection over the interval $\Delta t$. The density matrix will be acted on by jumps $\hat{J}_{ \pm}$ interspersed by unitary evolution due to Eq. (20). Because we do not have access to the microscopic details of photon arrival order nor photon arrival times, we must sum (integrate) over the final density matrices for all possible arrival orders (times) consistent with a particular photocount for the interval $\Delta t$. For a particular pair, this computation takes the form

$$
\begin{aligned}
\hat{\rho}(t+ & \left.+\Delta t ; C_{+}, C_{-}\right) \\
= & \sum_{\sigma} \int \cdots \int \hat{U}\left(t_{1}\right) \hat{J}_{\sigma_{1}} \cdots \hat{U}\left(t_{C}\right) \hat{J}_{\sigma_{C}} \hat{\rho}(t) \\
& \times \hat{J}_{\sigma_{C}}^{\dagger} \hat{U}^{\dagger}\left(t_{C}\right) \cdots \hat{J}_{\sigma_{1}}^{\dagger} \hat{U}^{\dagger}\left(t_{1}\right) \delta\left(\Delta t-\sum_{i=1}^{C} t_{i}\right) d t_{1} \cdots d t_{C},
\end{aligned}
$$

where $C=C_{+}+C_{-}, \sigma$ is summed over all permutations of click orders on the upper and lower arms of the Mach-Zehnder so that $\sigma_{i} \in\{+,-\}, t_{i}$ are the times of the clicks, and $U(t)=$ $\exp \left(-i g_{\mathrm{L}} \mu_{\mathrm{B}} \vec{B} \cdot \hat{\vec{F}} t\right){ }^{1}$

The calculation is simplified greatly by the fact that $\left[\hat{J}_{ \pm}, \hat{U}\left(t_{i}\right)\right]$ is of order $\epsilon^{3}$ or smaller (though there are order $\epsilon^{-1}$ such commutators, making their total effect of order $\left.\epsilon^{2}\right)$. We can therefore separate the jump operators (which commute with each other) from the unitary evolution operators. Because of the $\delta$ function, the product of $U\left(t_{1}\right) \ldots U\left(t_{C}\right)=U(\Delta t)$ and the integral over all $t_{i}$ gives $1 / C$ !. The sum over all permutations merely counts them, thereby giving a factor $\left(\begin{array}{c}C \\ C_{+}\end{array}\right)$. Thus, to order $\epsilon$,

$$
\begin{aligned}
\hat{\rho}\left(t+\Delta t ; C_{+}, C_{-}\right)= & \frac{1}{C_{+} ! C_{-} !} U(\Delta t) J_{+}^{C_{+}} J_{-}^{C_{-}} \hat{\rho}(t) \\
& \times J_{-}^{\dagger C_{-}} J_{+}^{\dagger C_{+}} U^{\dagger}(\Delta t) .
\end{aligned}
$$

The trace of the conditional density matrix Eq. (25) gives the relative probability of obtaining the associated photon count; we can use this probability distribution to determine the mean and variance of the photon counts. These are independent Gaussian distributions (to next-to-leading order), with

$$
\left\langle C_{ \pm}\right\rangle=V_{ \pm}=\frac{f \Delta t}{2}(1 \pm\langle\mathbf{G} \cdot \hat{\mathbf{N}}\rangle)
$$

that is, the mean equals the variance in the counts. The covariance between $C_{+}$and $C_{-}$vanishes to this order. Also, we note that the means reproduce the signal seen in Eq. (14). We are thus able to define the photoelectron counts as independent Gaussian processes with moments given by Eq. (26):

$$
C_{ \pm}=\frac{f}{2}(1 \pm\langle\mathbf{G} \cdot \hat{\mathbf{N}}\rangle) \Delta t+\sqrt{\frac{f}{2}(1 \pm\langle\mathbf{G} \cdot \hat{\mathbf{N}}\rangle)} \Delta W_{ \pm},
$$

where $\Delta W_{ \pm}$are independent Wiener increments, with

$$
\begin{aligned}
\left\langle\Delta W_{ \pm}^{2}\right\rangle_{\mathrm{E}} & =\Delta t, \\
\left\langle\Delta W_{+} \Delta W_{-}\right\rangle_{\mathrm{E}} & =0 .
\end{aligned}
$$

Here the subscript $E$ refers to the ensemble average (that is, over many experimental runs) rather than the usual expectation value. These Wiener increments are Gaussian random variables of zero mean that are functions of time. In the continuum limit, $\Delta \rightarrow d$, these equalities are exact without ensemble averaging.

Equation (27) is crucial to the derivation of the complete stochastic master equation. We find that, to order $\epsilon$, the products of jump operators appearing in Eq. (25) are given by

$J_{+}^{C_{+}} J_{-}^{C_{-}}=1-\frac{i}{2} \mathbf{G} \cdot \hat{\mathbf{N}}\left(f \Delta t-\sqrt{f} \Delta V_{+}\right)+\frac{1}{2} \sqrt{f} \Delta V_{-}$,

where $\Delta V$ are related to $\Delta W$ by

$$
\Delta V_{ \pm}=\frac{\Delta W_{+} \pm \Delta W_{-}}{\sqrt{2}} .
$$

\footnotetext{
${ }^{1}$ Here we assume that the external magnetic field varies slowly in the interval $\Delta t$.
} 
All that remains is to substitute Eq. (29) into Eq. (25); divide $\hat{\rho}(t+\Delta t)$ by its trace to normalize it, which cancels out the factors $C_{+} ! C_{-}$!; and then take the continuum limit. When carrying out these steps, care must be taken to keep terms up to $d V_{ \pm}^{2}$ because of the Itô rules [see Eq. (28)]. These last few steps yield

$$
\frac{d \hat{\rho}}{d t}=\frac{i}{\hbar}\left[\hat{\rho}, H^{\prime}\right]+\frac{\sqrt{f}}{2}(\mathbf{G} \cdot \hat{\mathbf{N}} \hat{\rho}+\hat{\rho} \mathbf{G} \cdot \hat{\mathbf{N}}-2\langle\mathbf{G} \cdot \hat{\mathbf{N}}\rangle \hat{\rho}) \xi_{-}(t),
$$

where the modified Hamiltonian $H^{\prime}$ is

$$
H^{\prime}=\hbar\left\{g_{\mathrm{L}} \mu_{\mathrm{B}} \vec{B} \cdot \hat{\vec{F}}+\frac{1}{2} \mathbf{G} \cdot \mathbf{N}\left[f+\sqrt{f} \xi_{+}(t)\right]\right\},
$$

and

$$
\xi_{ \pm}(t)=\frac{d V_{ \pm}}{d t}
$$

are uncorrelated Gaussian white-noise functions given by the continuum limit of Eq. (30).

Equations (31) and (32) succinctly encapsulate the full effects of the measurement scheme on the atoms. First, the Hamiltonian is modified by a steady, effective interaction resulting from Eq. (10), with the photon number operator replaced by a classical coherent flux. Shot noise in the photon flux leads to an additional random, but unitary, evolution. Finally, the second term in Eq. (31) is the nonunitary evolution induced by the measurement of $\mathbf{G} \cdot \hat{\mathbf{N}}$.

The validity of this master equation depends on the separation of scales in Eq. (21) remaining valid; in particular, if the photon flux becomes too large, the various commutators in the derivation are no longer negligible and must be accounted for. This situation is far more complex; however, as we see in the next section, such a situation will be far into a parameter region where the measurement rapidly causes dephasing of the spins.

\section{EXAMPLES}

We first examine a very simple application of this master equation to illustrate its salient features in an analytically closed form before moving on to the more experimentally relevant case of the measurement of a magnetic field. Namely, we will assume that there is no external field, $\vec{B}=0$, and coupling coefficients $G_{+}=-G_{-}=G$, with $G_{0}=0$. This means that the setup will measure only $\hat{F}_{z}$, i.e.,

$$
\mathbf{G} \cdot \hat{\mathbf{N}}=G \hat{F}_{z},
$$

and the total Hamiltonian is quantum nondemolition (QND) for the observable $\hat{F}_{z}$.

We can derive equations of motion for the observables of the system by multiplying Eq. (31) by the corresponding operators and taking the trace. Because of the large number of atoms involved, we assume that the Gaussian state ansatz will be valid; thus, only equations for the observables, their variances, and their covariances are needed. The chain rule of Itô calculus [resulting from Eq. (28)] must be used when considering the derivatives of the (co)variances, as they are nonlinear functions of the other variables. We obtain, for example, $d\left\langle\hat{F}_{z}\right\rangle / d t=G \sqrt{f} \xi_{-}(t) v_{z}$, where $v_{z}$ is the variance of $\left\langle\hat{F}_{z}\right\rangle$, and $\dot{v}_{z}=-f G^{2} v_{z}^{2}$, which quickly integrates to

$$
v_{z}(t)=\left(v_{z}(0)^{-1}+f G^{2} t\right)^{-1} .
$$

Note that determination of the mean value depends on the experimental record through $\xi_{-}(t)$, whereas the variance always decreases as the measurement continues. This should be unsurprising - continuous observation of $\hat{F}_{z}$ leads to greater certainty in its value. Over long times, the uncertainty can drop to a point where spin squeezing is achieved.

For the next example we include a dc magnetic field to be measured. We measure it by continuously observing the spin in a transverse direction: the atoms' Larmor precession frequency then reveals the field strength. We present the results of a numerical integration of the master equation over a single simulated experimental run. For concreteness we assume a condensate of $10^{4}$ atoms confined within a transverse spatial extent of $15 \mu \mathrm{m}$ in an ambient magnetic field of $1 \mathrm{mG}$ in the $y$ direction, and we apply an additional magnetic field $G f /\left(2 g_{\mathrm{L}} \mu_{\mathrm{B}}\right)$ in the negative $z$ direction. Since $G$ and $f$ are well-controlled experimental parameters this should be possible to a high degree of accuracy. This additional field cancels out the classical portion of the light-matter interaction, though the photon shot-noise fluctuations in Eq. (32) are still present. The probe is detuned by $2 \pi \times 150 \mathrm{MHz}$ below the $F=1 \rightarrow F^{\prime}=2\left(D_{1}\right)$ transition of ${ }^{87} \mathrm{Rb}$ and interacts with the condensate for a total measurement time of $100 \mathrm{~ms}$. The evolution of the condensate spin is shown for probe fluxes of $f=2 \times 10^{13} \mathrm{~s}^{-1}$ (Fig. 2) and $f=2 \times 10^{15} \mathrm{~s}^{-1}$ (Fig. 3). The measurement strength can best be characterized by the dimensionless ratio $G f / \nu_{\mathrm{L}}$, where $\nu_{\mathrm{L}}$ is the Larmor frequency. For the parameters listed, this ratio is 0.1 and 10 , respectively. In the former case, the free evolution of the atoms is not noticeably perturbed, yet the photocurrent signal unambiguously oscillates at the Larmor frequency. This will allow an accurate determination of the applied field. On the other hand, as the probe intensity is increased and the ratio

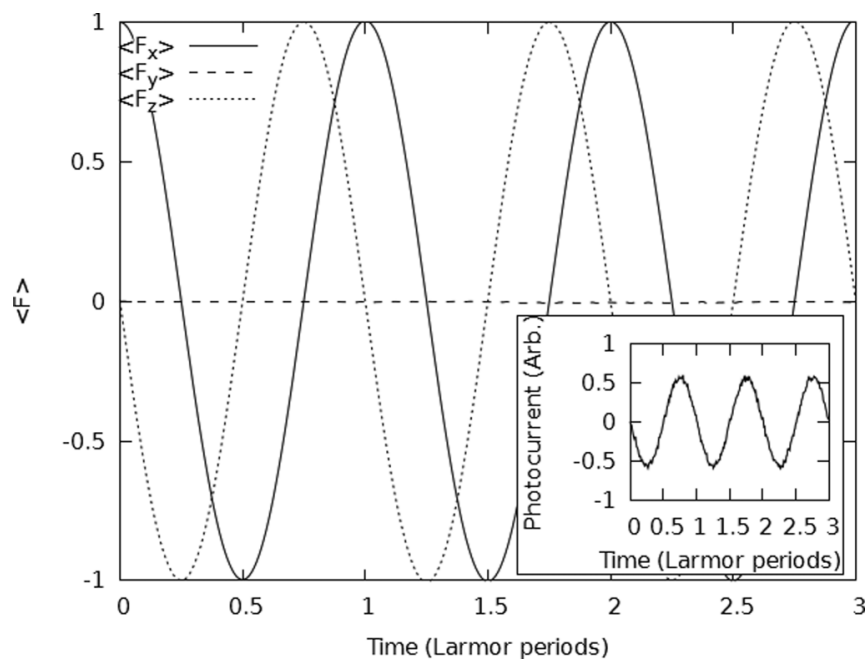

FIG. 2. The evolution of the condensate's mean spin along each axis for $f=2 \times 10^{13} \mathrm{~s}^{-1}$, normalized by atom number, for a single simulated experimental run. The optical detection of Larmor precession has very little effect on the free dynamics. Inset: The normalized photocurrent difference oscillating at the correct frequency. 


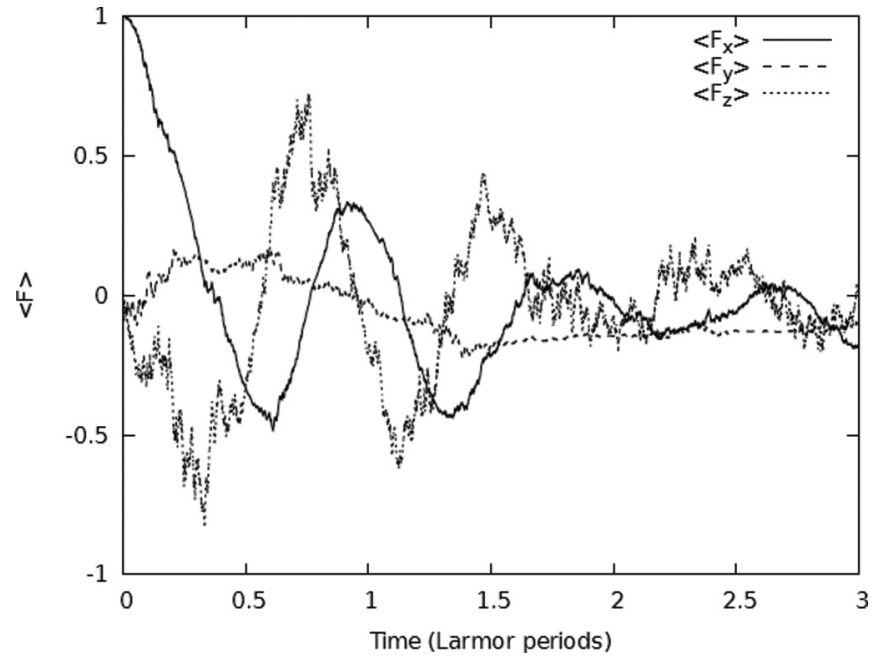

FIG. 3. The evolution of the condensate's mean spin projected along each axis for $f=2 \times 10^{15} \mathrm{~s}^{-1}$ for a single run. This stronger measurement causes rapid decay of the condensate's spin.

of the measurement strength to the Larmor frequency exceeds unity, the backaction-induced stochastic evolution overwhelms the free Larmor precession, resulting in a rapid decay of the transverse magnetization of the condensate in only a few oscillations. This crossover in behavior could be anticipated, as the light-atom interaction even in the absence of measurement dominates the precession of the atoms in the background field for such high measurement strengths.

\section{SUMMARY AND OUTLOOK}

We have provided a theoretical treatment of the quantum backaction due to the dispersive interaction between a spinor Bose-Einstein condensate and an off-resonant light field. In addition to being the basis for optical magnetometry using a Bose-Einstein condensate, this interaction has also been shown to be a versatile quantum interface for quantum information processing and state engineering [17].

Our treatment begins with a fairly general off-resonant light-atom interaction. The phase shift imprinted on the light provides information about the spin state of the atoms. We first derived the effects of measuring this phase shift on a condensate of identical spins. Next, we found a quantum jump operator characterizing the effects of measuring the passage of a single photon through the atomic ensemble. By then aggregating many such jumps along with evolution induced by a magnetic field, we found a master equation that governs the conditional evolution of the atomic spins under continuous measurement by light. This master equation reveals that, for sufficiently intense probe light, the spin state is significantly altered by measurement backaction.
Future additions to our model will include a description of spatially inhomogeneous spin textures in the condensate as well as an extension of the atomic manifold to include more varied systems such as multicomponent BECs. We also plan to include time dependence both of the magnetic field and of the optical measurement strength, e.g., stroboscopic measurements. In addition to understanding the potential sensitivity of condensate-based magnetic-field sensing, our formalism can also be applied to quantum-limited nondestructive imaging of magnetic textures in spinor condensates and the creation of novel many-body states via quantum nondemolition (QND) measurement [18].

\section{ACKNOWLEDGMENTS}

This work was supported by the Defense Advanced Research Project Agency (DARPA) QuASAR program through a grant from AFOSR, by the DARPA ORCHID program through a grant from the US Army Research Office, and by NSF. M.V. acknowledges support from the Alfred P. Sloan Foundation. The authors would also like to thank Carlo Samson and Chandra Raman of the Georgia Institute of Technology for useful input on additional experimental considerations.

\section{APPENDIX: DETAILS OF A SPECIFIC LIGHT-MATTER INTERACTION HAMILTONIAN}

As a concrete example of a light-matter Hamiltonian of the type in Eq. (10), we examine the case of circularly polarized light incident on a condensate of ${ }^{87} \mathrm{Rb}$ atoms. We consider only contributions from the $D_{1}$ virtual transitions between the ground-state $S_{1 / 2}$ and excited $P_{1 / 2}$ manifolds but add the effects of the virtual transitions from the $F=1$ to both the $F^{\prime}=1$ and 2 submanifolds. We find in the rotating wave approximation, and after adiabatically eliminating the excited atomic states,

$$
H_{I}=\frac{\hbar \Omega^{2}}{48 \Delta_{2}}\left[6 \hat{N}_{+}+(3+\delta) \hat{N}_{0}+(1+\delta) \hat{N}_{-}\right] \hat{b}^{\dagger} \hat{b} .
$$

Here the spin basis is in the direction of light propagation, $\Omega$ is the single-photon Rabi frequency, $\Delta_{2}$ is the detuning (possibly negative) for the $F=1 \rightarrow F^{\prime}=2$ transition, $\Delta_{1}=$ $\Delta_{2}+814.5 \mathrm{MHz}$ is the $F=1 \rightarrow F^{\prime}=1$ detuning, and $\delta$ is the experimentally adjustable ratio given by

$$
\delta=\frac{\Delta_{2}}{\Delta_{1}} .
$$

This parameter adds an element of tunability to the coupling strengths g, thereby allowing us to change the relative magnitudes of the different components of the signal given in Eq. (14).
[1] J. Taylor, P. Cappellaro, L. Childress, L. Jiang, D. Budker, P. Hemmer, A. Yacoby, R. Walsworth, and M. Lukin, Nature Physics 4, 810 (2008).

[2] G. Balasubramanian et al., Nature (London) 455, 648 (2008).

[3] J. Clarke, in SQUID Sensors: Fundamentals, Fabrication and Applications (Springer, New York, 1996), pp. 1-62.
[4] D. Budker and M. Romalis, Nature Phys. 3, 227 (2007).

[5] H. B. Dang, A. C. Maloof, and M. V. Romalis, Appl. Phys. Lett. 97, 15110 (2010).

[6] V. Petersen, L. B. Madsen, and K. Molmer, Phys. Rev. A 71, 012312 (2005). 
[7] M. Auzinsh, D. Budker, D. F. Kimball, S. M. Rochester, J. E. Stalnaker, A. O. Sushkov, and V. V. Yashchuk, Phys. Rev. Lett. 93, 173002 (2004).

[8] W. Wasilewski, K. Jensen, H. Krauter, J. J. Renema, M. V. Balabas, and E. S. Polzik, Phys. Rev. Lett. 104, 133601 (2010).

[9] M. Vengalattore, J. M. Higbie, S. R. Leslie, J. Guzman, L. E. Sadler, and D. M. Stamper-Kurn, Phys. Rev. Lett. 98, 200801 (2007).

[10] L. K. Thomsen, S. Mancini, and H. M. Wiseman, J. Phys. B 35, 4937 (2002).

[11] K. Zhang, L. Zhou, H. Y. Ling, H. Pu, and W. Zhang, Phys. Rev. A 83, 063624 (2011).

[12] I. Carusotto and E. J. Mueller, J. Phys. B 37, S115 (2004).
[13] J. M. Higbie, L. E. Sadler, S. Inouye, A. P. Chikkatur, S. R. Leslie, K. L. Moore, V. Savalli, and D. M. Stamper-Kurn, Phys. Rev. Lett. 95, 050401 (2005).

[14] D. Budker, W. Gawlik, D. F. Kimball, S. M. Rochester, V. Y. Yashchuk, and A. Weis, Rev. Mod. Phys. 74, 1153 (2002).

[15] A. Kuzmich, N. P. Bigelow, and L. Mandel, Europhys. Lett. 42, 481 (1998).

[16] C. W. Gardiner and M. J. Collett, Phys. Rev. A 31, 3761 (1985).

[17] K. Hammerer, A. S. Sorensen, and E. S. Polzik, Rev. Mod. Phys. 82, 1041 (2010).

[18] I. B. Mekhov and H. Ritsch, J. Phys. B 45, 102001 (2012). 